\title{
Epidural hematomas of the posterior cranial fossa
}

\author{
Edson Bor-Seng-Shu, M.D., Paulo Henrique Aguiar, M.D., \\ Ricardo Jose de Almeida Leme, M.D., Mauricio Mandel, \\ Almir Ferreira de Andrade, M.D., And Raul Marino JR., M.D. \\ Division of Neurosurgery, Hospital das Clinicas, São Paulo University Medical School, \\ São Paulo, Brazil
}

\begin{abstract}
Object. The authors present their experience in the management of posterior fossa epidural hematoma (PFEDH), which involved an aggressive diagnostic approach with the extensive use of head computerized tomography (CT) scanning.

Methods. The authors treated 43 cases of PFEDH in one of the largest health centers in Brazil. Diagnosis was established in all patients with the aid of CT scanning because the clinical manifestations were frequently nonspecific. Cases were stratified by clinical course, Glasgow Coma Scale score, and their radiological status. Based on clinical and radiological parameters the patients underwent surgical or conservative management.

Conclusions. Compared with outcomes reported in the available literature, good outcome was found in this series. This is primarily due to the broad use of CT scanning for diagnostic and observational purposes, which, in the authors' opinion, led to early diagnosis and prompt treatment.
\end{abstract}

\section{KEY WORDS • hematoma - posterior fossa - computerized tomography scanning}

Traumatic hematomas of the cranial posterior fossa occur much less frequently than supratentorial hematomas. ${ }^{1,7,13,19,22}$ They are, however, the most common traumatic space-occupying lesions of the posterior fossa ${ }^{18}$ and are a serious complication secondary to head injury. Clinically, symptoms may be silent and slow, but the associated deterioration is often sudden and quick becoming fatal if not promptly treated. ${ }^{4}$ Among conditions treated by neurosurgeons, PFEDH has always been considered rare and is associated with a difficult diagnosis and a high mortality rate.

Since the advent of CT scanning, the diagnosis of this lesion has been simplified and the prognosis of patients has improved. 5,6,8,12,14 There has been an increase in cases of PFEDH without mass effect treated conservatively with good results. ${ }^{10,11,15,16,21}$

\section{CLINICAL MATERIAL AND METHODS}

\section{Patient Population}

Between January 1986 and August 1999, 43 patients with an established diagnosis of PFEDH were admitted to the neurosurgical department of Hospital das Clinicas of the São Paulo University Medical School. The male/female ratio was 33:10. Patients ranged in age from 3 to 59 years (mean 18.3 years). The clinical signs and symptoms on admission are summarized in Table 1. All hematomas

Abbreviations used in this paper: $\mathrm{CT}=$ computerized tomography; GCS = Glasgow Coma Scale; PFEDH = posterior fossa epidural hematoma. were of traumatic origin. The most frequent causes were falls from height ( 26 cases), motor vehicle accidents (15 cases), and falls from a train in motion (two cases).

\section{Evaluation Protocol}

All patients underwent cranial CT scanning. Radiography was performed in 38 cases. The clinical course of traumatic PFEDH was classified according to the proposal of Hooper ${ }^{9}$ as acute, subacute, and chronic, with the onset of symptoms within the first 24 hours of trauma, from the 2 nd to the 7th day after trauma, and later, respectively (Table 2). The $\mathrm{GCS}^{20}$ was used to assess the level of consciousness in all patients (Table 3) and also to measure status in surviving patients to compare the surgical and the conservative managements. Associated traumatic lesions were identified by means of head CT scanning (Table 4). Long-term outcome was not assessed in these patients.

\section{Surgical and Conservative Treatments}

Forty patients underwent surgery and three conservative treatment. In patients who underwent surgical treatment, CT scans had revealed a mass effect due to hematoma, partial or total obliteration of the perimesencephalic cisterns, compression, and/or displacement of the fourth ventricle (Fig. 1). Minimal or small hematomas were surgically treated if associated with other intracranial traumatic lesions. In all surgical cases, the patient was placed prone to prevent development of air embolism. The standard approach in 40 cases was the wide suboccipital craniectomy. In cases in which the hematoma extended to the supratentorial occipital region, an occipital craniotomy 
E. Bor-Seng-Shu, et al.

TABLE 1

Clinical findings in 43 patients with PFEDH

\begin{tabular}{lc}
\hline \hline \multicolumn{1}{c}{ Signs \& Symptoms } & No. of Cases (\%) \\
\hline occipital, suboccipital, or retromastoid swelling & $36(84)$ \\
vomiting & $24(55.8)$ \\
headache & $24(55.8)$ \\
transient loss of consciousness & $18(41.9)$ \\
disturbance of consciousness & $16(37.2)$ \\
diplopia \& abducent paralysis & $7(16.3)$ \\
nuchal rigidity & $7(16.3)$ \\
otorrhagia & $4(9.3)$ \\
anisocoria & $2(4.7)$ \\
asymptomatic & $2(4.7)$ \\
\hline
\end{tabular}

was also performed when the supratentorial portion of the clot was large. Although feasible, the suboccipital craniotomy to approach the posterior fossa was not performed, even in children. Table 5 provides a summary of the GCS scores determined at the time a patient was stratified to the surgical or conservative treatment group. In the three patients receiving nonsurgical treatment, the decision to undertake conservative therapy was based on the clinical absence of brainstem compression or posterior fossa hypertension. These patients underwent careful observation of neurological status and control CT scanning (Fig. 2).

\section{RESULTS}

\section{Clinical Presentation}

The most important findings were occipital, suboccipital, or retromastoid swelling (36 cases), headache (24 cases), and vomiting (24 cases) (Table 1). Twenty-one patients were considered to harbor acute PFEDHs, 18 subacute lesions, and four chronic PFEDHs. On admission, a GCS score of 5 to 6 was documented in two patients, a score of 7 to 8 in three, a score of 9 to 12 in seven, and a score of 13 to 15 in 31 patients (Table 3 ).

\section{Radiological Findings}

Skull radiographs were obtained in 38 patients, in whom occipital fractures were detected in 30 (79\%). In the remaining eight cases and the five in which radiographs were not obtained, an occipital fracture was observed in six on CT scans or intraoperatively. In seven cases $(16.3 \%)$, we were unable to find evidence of an occipital fracture radiologically (on x-ray films or CT scans) or intraoperatively. Diastatic fractures, occipital linear fractures, or both were the most commonly encountered.

In four patients the PFEDHs extended into the supratentorial region; in one case the PFEDH coexisted with a

TABLE 2

Clinical course stratified by Hooper category for PFEDH

\begin{tabular}{cc}
\hline \hline Clinical course & No. of Cases $(\%)$ \\
\hline acute & $21(48.8)$ \\
subacute & $18(41.9)$ \\
chronic & $4(9.3)$
\end{tabular}

TABLE 3

Neurological status characterized by admission GCS score

\begin{tabular}{cc}
\hline \hline GCS Score & No. of Cases $(\%)$ \\
\hline $5-6$ & $2(46.5)$ \\
$7-8$ & $3(69.8)$ \\
$9-12$ & $7(16.3)$ \\
$13-15$ & $31(72.1)$ \\
\hline
\end{tabular}

supratentorial subdural hematoma. Unilateral PFEDHs were present in 30 patients (17 on the left and 13 on the right); bilateral hematomas were present in 13 patients.

\section{Associated Lesions}

In relation to the associated intracranial lesions coexisting with the PFEDHs, we found brain contusion in 12 cases, brain swelling in five, acute subdural hematomas in five, cerebellar contusion in two, subarachnoid hemorrhage in two, and posttraumatic hydrocephalus in nine cases (Table 4).

\section{Conservative Management}

In three patients in whom there were small hematomas not exerting mass effect as well as absence of other associated traumatic lesions, conservative therapy was elected. Admission GCS scores in these three patients were 15, and none died. At discharge, the patients were free of deficits and their GCS scores remained 15. Each of these patients was a child. Hospitalization was continued for at least 1 week until clinical and radiological deterioration had been excluded.

\section{Surgical Management}

Forty patients in whom the hematoma caused mass effect, even if the lesion was small or associated with other traumatic lesions, were treated surgically. The mortality rate in this group was $5 \%$. Two patients with acute PFEDH died despite prompt evacuation of the clot and supportive intensive care treatment. Eleven patients in whom the GCS score was less than 13 on admission improved after surgery, making an excellent recovery without neurological deficit. Twenty-five patients in whom the GCS score was 15 on admission and in whom large hematomas exerted mass effect underwent surgery, and their level of consciousness remained unchanged, without neurological deficit at discharge. Two patients

TABLE 4

Summary of trauma-induced lesions associated with PFEDH

\begin{tabular}{ll}
\hline \multicolumn{1}{c}{ Traumatic Lesions } & No. of Cases (\%) \\
\hline brain contusion & $12(27.9)$ \\
brain swelling & $05(11.6)$ \\
acute subdural hematoma & $03(7)$ \\
supratentorial & $02(4.7)$ \\
posterior fossa & $02(4.7)$ \\
cerebellar contusion & $02(4.7)$ \\
subarachnoid hemorrhage & $09(20.9)$ \\
posttraumatic hydrocephalus &
\end{tabular}




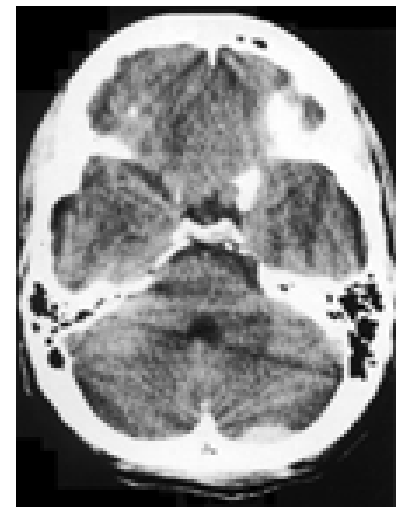

Fig. 1. Axial CT scan demonstrating a small PFEDH in a patient with a GCS score of 15 who underwent conservative treatment.

with acute PFEDH remained disabled after surgery. The bleeding source, responsible for the PFEDHs in 20 patients in whom the clinical course was acute, originated from the transverse sinus in 15 cases, oozing from meningeal vessels in three, and the sigmoid sinus in two. No bleeding source was found at surgery in cases involving the subacute or chronic clinical courses.

\section{DISCUSSION}

As expected in groups in which a lesion is caused by trauma, our population was composed primarily of young male individuals. Accordingly, early detection of the lesion is critical. The signs and symptoms were, in the majority of cases, nonspecific for acute PFEDH. In only a small percentage of cases could the diagnosis be established based on clinical findings. We believe that CT scanning should be conducted routinely in cases of head injury because in some patients with PFEDH the GCS score was 15 and the patients were asymptomatic. ${ }^{3}$ In addition, the only reason to visit the hospital might be the presence of an occipital, suboccipital, or retromastoid swelling, as seen in one of our patients. ${ }^{3}$ In our series, 31 patients presented with admission GCS scores of 13 to 15; however, in only 28 did this status remain unchanged while the management strategy was being decided. An early diagnosis is mandatory for good recovery, and some authors have suggested that CT scanning be conducted in all patients with occipital soft-tissue ecchymotic swelling and fracture of underlying occipital bone ${ }^{13}$ (Fig. 1). A

TABLE 5

Summary of treatment groups and associated GCS scores

\begin{tabular}{ccc}
\hline \hline & \multicolumn{2}{c}{ Treatment Group (\%) } \\
\cline { 2 - 3 } GCS Score & Surgery & Observation \\
\hline $13-15$ & $25(62.5)$ & $3(100) *$ \\
$9-12$ & $8(20)$ & \\
$7-8$ & $3(7.5)$ & \\
$5-6$ & $3(7.5)$ & \\
3 & $1(2.5)$ & \\
\hline
\end{tabular}

* Status in all three patients was represented by a GCS score of 15 .

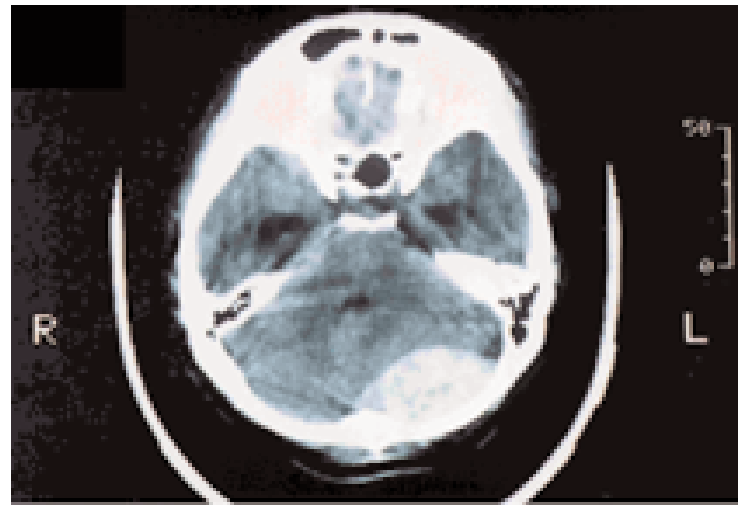

Fig. 2. Axial CT scan revealing partial mass effect of PFEDH and obliteration of the fourth ventricle. Note the presence of hydrocephalus.

high level of clinical suspicion, a prolonged period of clinical and radiological ${ }^{4}$ observation, and application of the broadest criteria for indication of head CT scanning ${ }^{3}$ are the key points to managing this situation. Control CT scanning performed within the first 24 hours plays a central role in this observational period because a slower course of the PFEDH or a delayed hematoma may be possible in the presence of occipital fractures.,11,17,21 $\mathrm{We}$ believe that radiological changes always occur earlier than clinical changes and should be monitored to predict the clinical progression. ${ }^{3,4}$

The incidence of bilateral hematomas was relatively high in our series $(30 \%)$. The bleeding source could be identified in virtually all acute cases $(95.2 \%)$, and despite the limited number of patients, the transverse sinus was consistently the main bleeding source in those cases (75\%), a finding comparable with those in the literature. ${ }^{4}$ The most common associated traumatic lesions in our series were brain contusions and posttraumatic hydrocephalus. The lesion can be classified as pure PFEDH if the hematoma is located within the boundary of foramen magnum and the transverse and sigmoid sinuses, and as mixed if it extends beyond the sinuses to the occipital area. $^{13}$

Deciding between conservative or surgical management is somewhat controversial. ${ }^{1,2,4,11,15-17}$ A GCS score of 15 and the exclusion of both mass effect and associated lesions were the criteria in this series to indicate conservative therapy. Bilateral suboccipital craniectomy was the standard approach in patients in whom the aforementioned criteria were not met. In $20 \%$ of the patients with acute PFEDH, a poor outcome was observed: two died and two remained neurologically disabled, results that call to mind the lethal potential of this entity, despite prompt treatment with all available resources. All patients in the subacute or chronic groups experienced an excellent outcome compared with the poorer prognosis (higher morbidity and mortality rates) found in the acute group; this is comparable with reports in the literature. ${ }^{4}$ In our series, the overall mortality rate of $4.7 \%$ can be considered to be a promising result when it is seen in comparison with those recently reported. . $^{2,4,13,14,17}$ We believe this is likely to be explained by the "aggressive" use of cranial CT scanning and consequent early diagnoses. Unfortunately, it 
was not possible to evaluate the long-term outcome in our patients. Future studies should be performed to address the long-term quality of life and the major factors limiting the resumption of daily activities in patients with traumatic PFEDH.

\section{CONCLUSIONS}

Patients with occipital trauma presenting with related symptoms or occipital fracture should undergo CT scanning and close observation. Once the diagnosis of a PFEDH is established, the hematoma must be evacuated immediately, except in special cases.

\section{References}

1. Ammirati M, Tomita T: Posterior fossa epidural hematoma during childhood. Neurosurgery 14:541-544, 1984

2. Boiten J: Epidural haematoma of the posterior fossa: good results after prompt diagnosis with CT. J Neurol Neurosurg Psychiatry 52:914-915, 1989

3. Bor-Seng-Shu E, Aguiar PH, Matushita H, et al: Actual asymptomatic epidural hematomas in childhood. Report of three cases. Childs Nerv Syst 13:605-607, 1997

4. Bozbuga M, Izgi N, Polat G, et al: Posterior fossa epidural hematomas: observations on a series of 73 cases. Neurosurg Rev 22:34-40, 1999

5. Bricolo AP, Pasut LM: Extradural hematoma: toward zero mortality. A prospective study. Neurosurgery 14:8-12, 1984

6. Ersahin Y, Mutluer S: Posterior fossa extradural hematomas in children. Pediatr Neurosurg 19:31-33, 1993

7. Gutierrez FA, McLone DG, Raimondi AJ: Physiopathology and a new treatment of chronic subdural hematoma in children. Childs Brain 5:216-232, 1979

8. Holzschuh M, Schuknecht B: Traumatic epidural haematomas of the posterior fossa: 20 new cases and a review of the literature since 1961. Br J Neurosurg 3:171-180, 1989

9. Hooper RS: Extradural haemorrhages of the posterior fossa. $\mathbf{B r}$ J Surg 42:19-26, 1954

10. Jamieson KG, Yelland JDN: Extradural hematoma. Report of 167 cases. J Neurosurg 29:13-23, 1968
11. Kawakami Y, Tamiya T, Tanimoto T, et al: Nonsurgical treatment of posterior fossa epidural hematoma. Pediatr Neurol 6: $112-118,1990$

12. Kushner MJ, Luken MG III: Posterior fossa epidural hematoma. A report of three cases diagnosed with computed tomography. Neuroradiology 24:169-171, 1983

13. Lui TN, Lee ST, Chang CN, et al: Epidural hematomas in the posterior cranial fossa. J Trauma 34:211-215, 1993

14. Neubauer UJ: Extradural haematoma of the posterior fossa. Twelve years experiences with CT-scan. Acta Neurochir 87: 105-111, 1987

15. Pang D, Horton JA, Herron JM, et al: Nonsurgical management of extradural hematomas in children. J Neurosurg 59: 958-971, 1983

16. Pozzati E, Tognetti F: Spontaneous healing of acute extradural hematomas: study of twenty-two cases. Neurosurgery 18: 696-700, 1986

17. Pozzati E, Tognetti F, Cavallo M, et al: Extradural haematomas of the posterior cranial fossa. Observations on a series of 32 consecutive cases treated after the introduction of computed tomography scanning. Surg Neurol 32:300-303, 1989

18. Roda JM, Giménez D, Pérez-Higueras A, et al: Posterior fossa epidural hematomas: a review and synthesis. Surg Neurol 19: 419-424, 1983

19. Sadik AR, Epstein FJ, Ransohoff J: Epidural hematoma of posterior fossa. NY State J Med 78:801-803, 1978

20. Teasdale G, Jennett B: Assessment of coma and impaired consciousness. A practical scale. Lancet 2:81-84, 1974

21. Wong CW: The CT criteria for conservative treatment-but under close clinical observation-of posterior fossa epidural haematomas. Acta Neurochir 126:124-127, 1994

22. Zuccarello M, Pardatscher K, Andrioli GC, et al: Epidural hematomas of posterior cranial fossa. Neurosurgery 8:434-437, 1981

Manuscript received October 16, 2003.

Accepted in final form December 18, 2003.

Address reprint requests to: Mauricio Mandel, Rua Dona Anton ia de Queiroz, 183 apto 804, Consolacão, São Paulo - SP Brazil 01307-010. email: mandelfmusp@ hotmail.com. 